EUROPEAN JOURNAL OF PURE AND APPLIED MATHEMATICS

Vol. 11, No. 1, 2018, 260-283

ISSN 1307-5543 - www.ejpam.com

Published by New York Business Global

\title{
Flows Spectrum on Closed Trio of Contours with Uniform Load
}

\author{
Alexander P.Buslaev ${ }^{1,2, *}$, Alexander G.Tatashev ${ }^{2,1}$, Marina V.Yashina ${ }^{2,1}$ \\ 1 Department of Higher Mathematics, Automobile Faculty, Moscow Automobile and Road \\ Construction State Technical University (MADI), Moscow, Russia \\ 2 Department of Mathematical Cybernetics and IT, Faculty of Information Technology, \\ Moscow Technical University of Communications and Informatics, Moscow, Russia
}

\begin{abstract}
Considered dynamical system is a flow of clusters with the same length $l$ on contours of unit length connected in polar-remote points into closed chain. When clusters move through common node, the left-priority rule of competition resolution works.

In the paper it is shown that, in the case of chain containing three contours, the dynamical system has a spectrum of velocity and mode periodicity consisted of not more than two components.

Spectrum distribution in dependence on load $l$ is developed. Hypotheses on discrete spectrum in the case of arbitrary number of contours are formulated.
\end{abstract}

2010 Mathematics Subject Classifications: 47J10, 05C21, 11J06, 76B75

Key Words and Phrases: Dynamical System, Spectrum, Self-organization, Contour Graph, Collapse, Cluster Model

\section{Introduction}

\subsection{System description}

We consider a closed chain of 3 contours - circles of unit length $\left(C_{1}, C_{2}, C_{3}\right)$. On each contour there is a standard coordinate system defined from 0 to 1 in counterclockwise direction. The coordinates of nodes - common points of neighboring contours $\left(C_{1}, C_{2}\right)$, $\left(C_{2}, C_{3}\right),\left(C_{3}, C_{1}\right)$ are equal to $(0,1 / 2),(0,1 / 2),(0,1 / 2)$ correspondingly. On all contours $C_{i}, i=1,2,3$, clusters of length $l$ move counterclockwise and with velocity 1 equal to the complete circle per time unit. A system state at time $t$ is a vector

$$
\left(\alpha_{1}(t), \alpha_{2}(t), \alpha_{3}(t)\right)
$$

where $\alpha_{1}(t), \alpha_{2}(t), \alpha_{3}(t)$ are coordinates of the leading points of clusters $C_{1}, C_{2}$, and $C_{3}$ correspondingly.

\footnotetext{
${ }^{*}$ Corresponding author.

Email addresses: apal2006@yandex.ru (A.P. Buslaev), a-tatashev@yandex.ru (A.G. Tatashev ), yashinamv14@gmail.com (M.V. Yashina), 
The trailing points of clusters $C_{1}, C_{2}$ and $C_{3}$, at the moment $t$, are located in points with coordinates $\alpha_{1}(t)-l, \alpha_{2}(t)-l, \alpha_{3}(t)-l$ respectively (subtraction by modulo 1 ).

The system state is called admissible state, if no one node is covered by more than one cluster. The delay of cluster movement occurs when the cluster approaches the node at the time that cluster on adjacent contour covers this node. If two clusters approach the same node simultaneously, then there is a competition. For the dynamical system it needs to determinate a rule of competition resolution. After a result of conflict resolution one of these clusters is delayed the node, and another cluster begins to pass the node.

Let us define the left-priority conflict resolution rule that means the following. If the conflict occurs between the clusters of contours $C_{i}$ and $C_{i+1}$, (addition modulo 3), then the cluster on contour $C_{i}, i=1,2,3$, moves though the common node (advantage over priority), and cluster on contour $C_{i+1}$ stops.

Periodic spectral point is called the admissible state $S_{0}=\left(\alpha_{1}(0), \alpha_{2}(0), \alpha_{3}(0)\right)$, for which there exist the minimal time values $T^{* *} \geq 1, T^{*} \geq 0$, such that $\forall t \geq T^{*}$

$$
\alpha_{1}\left(t+T^{* *}\right) \equiv \alpha_{1}(t), \alpha_{2}\left(t+T^{* *}\right) \equiv \alpha_{2}(t), \alpha_{3}\left(t+T^{* *}\right) \equiv \alpha_{3}(t) .
$$

Collapse (congestion) is a mode of dynamical system where all clusters do not have the capability of movement [2]. The dynamical system is in the free movement state at the time $t_{0}$, if at any time $t \geq t_{0}$ all clusters move without delays, [4]. We say that self-organization of the dynamical system is a property of the system such that it should result in free movement state over finite time from any admissible initial state, [1].

\section{Movement and collapse}

2.1. Collapse, $l>\frac{1}{2}$

Proposition 1. If cluster length

$$
l>\frac{1}{2}
$$

then for any admissible initial state the dynamical system results in the collapse state no later than through $1 / 2$ time units.

Proof.

From (2) we have that at each time unit any cluster covers at least one node. As the number of contours is equal to nodes number, then for any system state no cluster can cover two nodes simultaneously. And, therefore, the cluster approaching the node can not cross the node. Given that a moving cluster approaches one of the nodes no more than over $1 / 2$ time units, the proposition is proved.

\subsection{Movement (life), $l<\frac{1}{2}$}

Proposition 2. If $l<\frac{1}{2}$, then the instantaneous velocity of each cluster and instantaneous average velocity of the system are strictly separated from zero. 
Proof. If the condition is satisfied, then at any time unit at least one of three clusters is moving. Hence the instantaneous velocity of the system is not less than $1 / 3$. On the other hand, each cluster either moves without conflicts, or after a delay equals not more than $l$ time units at least $1 / 2$ moves without delay. Thus each cluster makes a turn during a finite time.

\subsection{System velocity}

The basic studied characteristic of the system is the average cluster velocity, determined by the limit $\lim _{T \rightarrow \infty} \frac{V(T)}{3 T}$, where $V(T)$ is the total distance such that all clusters passed during the time interval $(0, T)$. There arises the problem of the existence of velocity and periodic spectra. Since the system is deterministic and, as it will be shown by direct verification, for any initial state of the system, the system states are periodically repeating, starting from some finite time unit, then the limit exists and thus the average system velocity is determined.

Suppose that the initial state of the system is such that the average cluster velocity is less than 1. Cluster delays can occur at the node located on the left, at the point with the coordinate $\frac{1}{2}$, and at the node located on the right, at the point with coordinate 0 . In the first case, the cluster delay is ending when the coordinate of the leading point of cluster on the left contour takes the value $l$. In the second case, the cluster delay is ending when the coordinate of the leading point of cluster on right contour takes the value $\frac{1}{2}+l$. Then we can reduce the study of the spectrum of possible values of velocity to the consideration of behavior systems for two one-parameter sets of initial states.

Proposition 3. If the state

$$
\left(\alpha_{1}(0), \quad \frac{1}{2}, \quad \alpha_{3}(0)\right), \quad 0<\alpha_{1}(0)<l<\frac{1}{2}
$$

is periodic spectral point, then the state $\left(l, \frac{1}{2}, \quad \alpha_{3}(0)+l-\alpha_{1}(0)=\alpha_{30}\right)$ is also periodic spectral point.

If the state

$$
\left(\alpha_{1}(0), \quad 0, \quad \alpha_{3}(0)\right), \quad \frac{1}{2}<\alpha_{3}(0)<\frac{1}{2}+l
$$

is periodic spectral point, then the state $\left(\alpha_{10}=\alpha_{1}(0)+\frac{1}{2}+l-\alpha_{3}(0), \quad 0, \frac{1}{2}+l\right)$ is also periodic spectral point.

Proof. We suppose that at time $t=0$ the system is in the state

$$
\left(\alpha_{1}(0), \frac{1}{2}, \alpha_{3}(0)\right), 0<\alpha_{1}(0)<l<\frac{1}{2} .
$$

Then during the time interval $t \in\left(0, \frac{1}{2}+l-\alpha_{1}(0)\right)$ the cluster of contour $C_{2}$ does not move, but clusters of contours $C_{1} C_{3}$ are moving. 
Thus, at the moment $\frac{1}{2}+l-\alpha_{1}(0)$ the system is in the state

$$
\left(\alpha_{1}\left(t_{0}\right)+\frac{1}{2}+l-\gamma_{0}, 0, \frac{1}{2}+l\right) .
$$

Similarly, we consider the case when at the moment 0 the system is in the state

$$
\left(\alpha_{1}(0), 0, \alpha_{3}(0)\right), \frac{1}{2}<\alpha_{3}(0)<\frac{1}{2}+l .
$$

Proposition 3 has been proved.

Thus, if the cluster velocity is not equal to 1 , then the system approaches to one of the two states $\left(l, \frac{1}{2}, \alpha_{30}\right), 0 \leq \alpha_{30} \leq 1$, Fig. 1 , or $\left(\alpha_{10}, \frac{1}{2}, \frac{1}{2}+l\right), 0 \leq \alpha_{10} \leq 1$, Fig.2, up to a shift, during a finite time



Figure 1: System state $l, 1 / 2, \alpha_{30}$

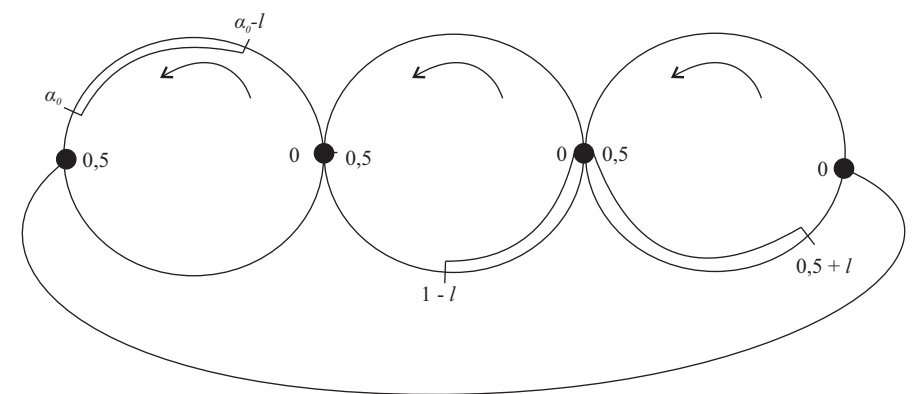

Figure 2: System state $\alpha_{10}, 1 / 2,1 / 2+l$

\subsection{Potential of delay and properties}

Let us denote

$$
\begin{aligned}
& \beta_{i}^{+}(t)=\left(\alpha_{i+1}(t)-\alpha_{i}(t)\right)_{\bmod (1)}, \\
& \beta_{i}^{-}(t)=\left(\alpha_{i}(t)-\alpha_{i+1}(t)\right)_{\bmod (1)},
\end{aligned}
$$


where the indices are computed by modulo 3. Let $\theta(t)$ be Heaviside function, $\psi(t)=$ $(\theta(t-1 / 2) \theta(l+1 / 2-t))$ Suppose that the coordinates of the leading points of clusters on adjacent contours $C_{i} C_{i+1}, i=1,2,3$, at time $t_{0}$ satisfy the relation

$$
\frac{1}{2} \leq \beta_{i}^{-}\left(t_{0}\right)<\frac{1}{2}+l
$$

Then at the time moment $t=\frac{1}{2}-\alpha_{i+1}\left(t_{0}\right)$ the cluster delay of contour $C_{i+1}$ begins, if the delay does not occur earlier. We define potential delay at the moment $t_{0}$ of cluster of contour $C_{i+1}$ relative of the cluster of contour $C_{i}$ the value equal to

$$
h_{i+1, i}\left(t_{0}\right)=\frac{1}{2}+l-\beta_{i}^{-}\left(t_{0}\right),
$$

if condition (3) fulfils, and $h_{i+1, i}\left(t_{0}\right)=0$, if condition (5) does not fulfill, $i=1,2,3$.

If the coordinates of the leading points of the clusters on neighbor contours $C_{i}$ and $C_{i-1}, i=1,2,3$, at time unit $t_{0}$ satisfy the relation

$$
\frac{1}{2}<\beta_{i-1}^{+}\left(t_{0}\right)<\frac{1}{2}+l
$$

then at the moment $t=\frac{1}{2}-\alpha_{i-1}\left(t_{0}\right)$ the delay of the cluster on contour $C_{i+1}$ will begin, if the delay does not begin earlier.

We shall say that potential delay at the time $t_{0}$ of the cluster on $C_{i-1}$ with respect to cluster of the contour $C_{i}$, is the value equal to

$$
h_{i-1, i}\left(t_{0}\right)=\frac{1}{2}+l-\beta_{i-1}^{+}\left(t_{0}\right),
$$

if condition (6) fulfils, and

$$
h_{i-1, i}\left(t_{0}\right)=0,
$$

if condition (6) does not fulfill, $i=1,2,3$. Thus,

$$
\begin{gathered}
h_{i+1, i}\left(t_{0}\right)=\left(\frac{1}{2}+l-\beta_{i}^{-}\left(t_{0}\right)\right) \psi\left(\beta_{i}^{-}\left(t_{0}\right)\right), \\
h_{i-1, i}\left(t_{0}\right)=\left(\frac{1}{2}+l-\beta_{i-1}^{+}\left(t_{0}\right)\right) \psi\left(\beta_{i-1}^{+}\left(t_{0}\right)\right) .
\end{gathered}
$$

We say that delay potential is a function of time

$$
\begin{aligned}
& H(t)=h_{1,2}(t)+h_{2,1}(t)+h_{2,3}(t)+h_{3,2}(t)+h_{3,1}(t)+h_{1,3}(t)= \\
= & \sum_{i=1}^{i=3}\left(\left(1 / 2+l-\beta_{i}^{-}(t)\right) \psi\left(\beta_{i}^{-}(t)\right)+\left(1 / 2+l-\beta_{i}^{+}\left(t_{0}\right) \psi\left(\beta_{i}^{+}(t)\right)\right)\right) .
\end{aligned}
$$

Proposition 4. Delay potential is non-negative piecewise linear function of time, and its derivative has one of the following values $-2,-1,0$ or 1 . 
Proof.

Proposition 4 follows from the fact that the velocity of changes of potential delay for any cluster relative to neighbor cluster at each time unit has one of values $-1,0$ or 1 .

And, in a closed chain of 3 contours, a delay of not more than one cluster takes place simultaneously, and this cluster can have non-zero potential delays relative to one or two clusters.

Proposition 5. Delay potential is a nonincreasing function of time.

Proof. Suppose at some time unit $t$ the function $H(t)$ increases.

This is possible only in the case such that at least one term on the right-hand side of equation (3) is increasing function. Suppose the term $h_{i_{0}, i_{0}+1}(t)$ increases (index addition by modulo $n$ ). We note the term can increase only with velocity 1 . This term can increase only if the cluster on contour $C_{i_{0}}$ moves, and the cluster on contour $C_{i_{0}+1}$ does not move. If cluster on contour $C_{i_{0}+1}$ is near a node being common with contour $C_{i_{0}}$, then term $h_{i_{0}, i_{0}+1}(t)$ needs to decrease, but not increase. Hence cluster on contour $C_{i_{0}+1}$ does not move near node being common for contours $C_{i_{0}+1}$ and $C_{i_{0}+2}$, and cluster on contour $C_{i_{0}+2}$ passes the node. But the value $h_{i_{0}+1, i_{0}+2}(t)$ at time $t$ decreases with velocity 1 .

Similarly, it is proved that if at a given time $t_{0}$ the term $h_{i_{0}, i_{0}-1}\left(t_{0}\right)$ increases, then at $t_{0}$ term $h_{i_{0}-1, i_{0}-2}\left(t_{0}\right)$ decreases. Thus each increasing term in the sum on the right-hand side of equality (3) corresponds to the decreasing term with the same velocity, and different increasing terms are associated with various decreasing terms.

Thus Proposition 5 has been proved.

Proposition 6. For any $i$ the following equation holds

$$
h_{i+1, i}(t) h_{i, i+1}(t)=0
$$

Proof. In accordance with definitions (5)-(6) the identity

$$
\beta_{i}^{-}(t)+\beta_{i}^{+}(t)=1
$$

holds.

Proposition 6 is proved.

Proposition 7. The system at the time $t_{0}$ is in the free movement state if and only if $H\left(t_{0}\right)=0$.

Proof. If $H\left(t_{0}\right)=0$, then not for any $i=1,2,3$ the condition (5) or (6) fulfils, and therefore, after the time $t_{0}$ there are not delays.

If $H\left(t_{0}\right) \neq 0$, then for some $i=1,2,3$ the condition (5) or (6) fulfils, and therefore, after the time $t_{0}$ there is at least one delay in cluster movement.

\subsection{Concept of closed clusters group}

We introduce the following definitions. Clusters at the time unit $t$ form closed clusters group if for any $i, i=1,2,3$ either $h_{j, j+1}(t)+h_{j+1, j}(t)>0$, or $\beta_{j}^{-}(t)=\frac{1}{2}+l$, or $\beta_{j}^{+}(t)=\frac{1}{2}+l$ is fulfilled. 
Note that if equality $\beta_{i}^{-}\left(t_{0}\right)=\frac{1}{2}+l$ is true, then if in interval of time $t \in\left(t_{0}, t_{0}+\varepsilon\right)$ cluster on contour $C_{i}$ does not move, and cluster on contour $C_{i+1}$ moves, then for any arbitrarily small $\varepsilon>0$ the value $h_{i+1, i}\left(t_{0}+\varepsilon\right)$ is positive. Analogically, equality $\beta_{i}^{+}\left(t_{0}\right)=$ $\frac{1}{2}+l$ means that if in interval of time $t \in\left(t_{0}, t_{0}+\varepsilon\right)$ cluster on contour $C_{i}$ moves, and cluster on contour $C_{i+1}$ does not move, then for any arbitrarily small $\varepsilon>0$ the value $h_{i, i+1}\left(t_{0}+\varepsilon\right)$ is positive.

Clusters $C_{i}, \ldots, C_{i+k-1}$ form at time unit $t$ closed group of left-type, if for any $j$, $j=1, \ldots, i+k-2$ either $h_{j+1, j}(t)>0$ (addition modulo 3 ), nor $\beta_{j}^{-}(t)=\frac{1}{2}+l$. Clusters form at time unit $t$ closed group of right-type, if for any $j, j=1, \ldots, i+k-2$ either $h_{j, j+1}(t)>0$, nor $\beta_{j}^{+}(t)=\frac{1}{2}+l$. We call closed group by closed of mixed type, if it is not neither an open left-group nor an open group of the right-type.

Note that in the case of left-type group the cluster can not delay at node located right from it. And in the case of right-type group the cluster can not delay at node located left from it.

Proposition 8. Suppose that from some time unit $t_{0}$ the delay potential is positive and does not change

$$
H(t) \equiv H\left(t_{0}\right)>0, t \geq t_{0}
$$

Then clusters form closed group left- or right-type for $t>t_{0}$.

Proof. As $H\left(t_{0}\right)>0$, then the system at time $t_{0}$ is not in the free movement state.

Suppose that in the time interval $\left(t_{0}, t_{1}\right)$ all clusters move, and at the moment $t_{1}$ the delay of cluster on contour $C_{i}$ begins near the node common with contour $C_{i+1}$, then $h_{i, i+1}\left(t_{0}\right)=h_{i, i+1}\left(t_{1}\right)>0$ and term $h_{i, i+1}$ decreases in the time interval $\left(t_{1}, t_{2}\right)$, at the same time the value $h_{i+1, i}$ is equal to 0 in this time interval. The values $h_{i-1, i+1}$ and $h_{i+1, i-1}$ in interval $\left(t_{1}, t_{2}\right)$ don't change, because in this interval the clusters on contours $C_{i-1}$ and $C_{i+1}$ move.

If the function $H(t)$ does not decrease in the interval $\left(t_{1}, t_{2}\right)$, then one term of $h_{i-1, i+1}$ needs to increase, at the same time another term is equal to 0 . As cluster on contour $C_{i}$ does not move, then term $h_{i-1, i}$ needs to increase. If in some time unit $t_{3}\left(t_{1} \leq t_{3}<t_{2}\right)$ the equality $\beta_{i-1}^{+}\left(t_{3}\right)=\frac{1}{2}$ fulfils, then in time $t_{3}$ the value of $h_{i-1, i}$ abruptly decreases from $l$ to 0 , and value $h_{i, i-1}$ with jump increases from 0 to $l$. In time interval $\left(t_{2}, t_{3}\right)$ there will decrease both term $h_{i, i+1}$ and term $h_{i-1, i}$. So the value $H(t)$ will decrease. If the value $\beta_{i-1}^{+}$does not achieve the value $1 / 2$ in the interval $\left(t_{1}, t_{2}\right)$ (it is equivalent that $h_{i, i-1}$ does not achieve the value $l$ ), then the following relations hold

$$
\begin{gathered}
h_{i, i+1}\left(t_{2}\right)=h_{i+1, i}\left(t_{2}\right)=h_{i, i-1}\left(t_{2}\right)=0, \\
h_{i-1, i}\left(t_{2}\right)>0 .
\end{gathered}
$$

If $h_{i+1, i}\left(t_{0}\right)>0$, then at time $t_{0}$ the clusters form closed group of right-type. If $h_{i-1, i+1}\left(t_{0}\right)>$ 0 , then $h_{i-1, i+1}\left(t_{2}\right)>0, h_{i+1, i-1}\left(t_{0}\right)=h_{i+1, i-1}\left(t_{0}\right)>0$. Therefore, at some time unit $t_{4}>t_{2}$, the delay of cluster on contour $C_{i-1}$ begins near node common with contour $C_{i}$, or near node common with contour $C_{i+1}$. In both cases after the time $t_{2}$ both terms 
$h_{i-1, i+1}$ and $h_{i+1, i-1}$ will decrease. Thus, the value $H$ will decrease. If

$$
h_{i-1, i+1}\left(t_{0}\right)=h_{i-1, i+1}\left(t_{2}\right)=h_{i+1, i-1}\left(t_{0}\right)=h_{i+1, i-1}\left(t_{2}\right)=0,
$$

then in some time unit a delay of cluster on contour $C_{i-1}$ will begin near node common with contour $C_{i}$. Thus immediately after time $t_{5}$, the potential delay $h_{i-1, i}$ will decrease, and in order to the delay potential $H$ does not decrease, it is necessary that the value $h_{i+1, i-1}$ increases. As $h_{i+1, i-1}\left(t_{0}\right)=h_{i+1, i-1}\left(t_{5}\right)=0$, then the condition

$$
\beta_{i+1}^{+}\left(t_{0}\right)=\beta_{i+1}^{+}\left(t_{5}\right)=\frac{1}{2}+l
$$

needs to hold, and so at time $t_{0}$ the clusters form the group of right-type.

Analogically it is proved that if at time $t_{1}$ the delay of cluster on contour $C_{i}$ begins near the common node with the contour $C_{i-1}$, then at time $t_{0}$ the clusters form a group of left-type.

The Proposition 8 has been proved.

\section{Self-organization as a simple spectrum of a dynamical system}

Theorem 1. For a closed chain of 3 contours the sufficient condition for selforganization is the following condition

$$
l \leq \frac{1}{6}
$$

From Proposition 5 we have that delay potential is a nonincreasing function of time. From Proposition 8 , it is true, that either clusters from some moment $t_{0}$, or there are time intervals such that clusters are delayed and delay potential decrease, and, in this case, the delay potential will reach the value 0 for a finite time. Then consequently, the system results in free movement state.

But in the case $l<1 / 6$, clusters can not form a closed group. And in the case $l=1 / 6$ clusters can form a closed group, only if the delay potential is 0 .

Hence Theorem 1 is true.

\section{Case of a multiple spectrum $\left(\frac{1}{6}<l<\frac{1}{2}\right)$} Then

Proposition 9. Suppose $\frac{1}{6}<l<\frac{1}{2}$ and at time $t_{0}$ clusters form left- or right type.

$$
H\left(t_{0}\right)=3 l-\frac{1}{2} .
$$

Proof.

Let it be left-type group. For right-type group the proof is analogically.

We have

$$
H\left(t_{0}\right)=h_{13}\left(t_{0}\right)+h_{21}\left(t_{0}\right)+h_{32}\left(t_{0}\right)=
$$




$$
=\frac{3}{2}+3 l-\beta_{3}^{-}\left(t_{0}\right)-\beta_{2}^{-}\left(t_{0}\right)-\beta_{1}^{-}\left(t_{0}\right) .
$$

As

$$
\frac{1}{2} \leq \beta_{i}^{-}\left(t_{0}\right) \leq \frac{1}{2}+l, i=1,2,3
$$

then

$$
\begin{gathered}
\beta_{1}^{-}\left(t_{0}\right)+\beta_{2}^{-}\left(t_{0}\right)+\beta_{3}^{-}\left(t_{0}\right)= \\
=\left(\alpha_{2}\left(t_{0}\right)-\alpha_{1}\left(t_{0}\right)\right)_{\bmod (1)}+\left(\alpha_{3}\left(t_{0}\right)-\alpha_{2}\left(t_{0}\right)\right)_{\bmod (1)}+ \\
+\left(\alpha_{2}\left(t_{0}\right)-\alpha_{3}\left(t_{0}\right)\right)_{\bmod (1)}+\left(\alpha_{3}\left(t_{0}\right)-\alpha_{1}\left(t_{0}\right)\right)_{\bmod (1)}=2 .
\end{gathered}
$$

Proposition 10. Let $1 / 6<l \leq 1 / 2$ and the system does not result in free movement state over finite time. Then from some time unit $t_{0}$ clusters form a closed group of leftor right-type. And after time unit $t_{0}$, the vector state of the system is cyclically shifted one position to the right over time interval $\frac{1}{2}+l$ (in the case of left-type group), or one position to the left (in the case of right-type group). Also for this interval the total delay of clusters

$$
H(t)=3 l-1 / 2=H\left(t_{0}\right),
$$

and the clusters average velocity equals

$$
4 /(3+6 l)
$$

Proof. According to condition of Proposition 10 the system does not result in free movement state, then we consider with taking to account the Proposition 3 the system behavior with initial condition

$$
\left(l, \frac{1}{2}, \alpha_{3,0}\right), 0 \leq \alpha_{3,0}<1 .
$$

The case of initial condition

$$
\left(\alpha_{1,0}, 0, \frac{1}{2}+l\right), 0 \leq \alpha_{1,0}<1
$$

is considered analogically.

At initial time unit one of the following conditions fulfils:

$$
\begin{gathered}
0 \leq \alpha_{, 0}<l \\
l \leq \alpha_{3,0} \leq \frac{1}{2} \\
\frac{1}{2}<\alpha_{3,0}<\frac{1}{2}+l, l<\frac{1}{4},
\end{gathered}
$$




$$
\begin{gathered}
\frac{1}{2}+l \leq \alpha_{3,0} \leq 1-l, l \leq \frac{1}{4}, \\
1-l<\alpha_{3,0}<\frac{1}{2}+2 l, l<\frac{1}{4}, \\
\frac{1}{2}+2 l \leq \alpha_{3,0}<1, l<\frac{1}{4}, \\
\frac{1}{2}<\alpha_{3,0} \leq 1-l, l \geq \frac{1}{4} \\
1-l<\alpha_{3,0}<\frac{1}{2}+l, l>\frac{1}{4}, \\
\frac{1}{2}+l<\alpha_{30}<1, l \geq \frac{1}{4} .
\end{gathered}
$$

For any initial state belonging the considered one-parameter family we have

$$
h_{1,2}(0)=h_{2,1}(0)=0 .
$$

Each of cases $(13)-(21)$ is characterized by which values $h_{1,3}(0), h_{3,1}(0)=0, h_{2,3}(0)$, $h_{3,2}(0)$ are nonzero.

We describe the system behavior at the fulfilling of inequalities (13)-(21).

We will show below that if condition (14) is satisfied, the system is in free movement state at the initial time. In this case, the delay potential $H(t)$ is equal to 0 at any time. If conditions (13), (15), (19), (20) fulfill, the system results in free movement state over finite time. The delay potential $H(t)$ is a piecewise-linear nonincreasing function equal to 0 over some finite time.

If conditions (16), (17), (18), (21) hold, then beginning from finite time unit the system states periodically repeat, and average cluster velocity equals

$$
v=\frac{4}{3+6 l} \text {. }
$$

At fulfilling of conditions (16), (17), (21), cluster delays are arising cyclically in the following order: delay of cluster $C_{1}$ with the duration $2 l-\alpha_{30}+\frac{1}{2}$, delay of cluster $C_{3}$ with the duration $\alpha_{3,0}+l-1$, delay of cluster $C_{2}$ with the duration $2 l-\alpha_{3,0}+\frac{1}{2}$, delay of cluster $C_{1}$ with the duration $\alpha_{3,0}+l-1$, delay of cluster $C_{3}$ with the duration $2 l-\alpha_{3,0}+\frac{1}{2}$, delay of cluster $C_{2}$ with the duration $\alpha_{3,0}+l-1$.

Then, after the end of the period, the system results in a state $\left(\frac{1}{2}, \quad \alpha_{3,0}^{\prime}, \quad l\right)$ such that its vector is obtained from initial state vector by cyclic shift of one position to the right and substitution $\alpha_{3,0}$ to $\alpha_{3,0}^{\prime}, \alpha_{3,0}+\alpha_{3,0}^{\prime}=\frac{3}{2}+l$. During the following part of the period 
the clusters are delayed in the same order, while the delay times are equal to $\alpha_{3,0}^{\prime}+l-1$ $2 l-\alpha_{3,0}+\frac{1}{2}$.

At fulfilling of conditions (14), clusters are delayed in the period the following order: cluster delay $C_{3}$, cluster delay $C_{1}$, cluster delay $C_{2}$, while the duration of each of these delays is equal to $3 l-1$.

Delay potential at fulfilling of conditions (16), (17), (21) has constant value equal to $3 l-1$. At fulfilling of conditions (14) delay potential is piece-linear function such that its values do not change from some time and equal to $3 l-1$, if $\frac{1}{2}+2 l \leq \alpha_{3,0}<1$, and equal to $3 l-1$ from initial time, if $\alpha_{3,0}=\frac{1}{2}+2 l<1$.

As we will show below the behavior of the system with the initial state $\left(l, \frac{1}{2}, \alpha_{30}\right)$, $0 \leq \alpha_{30}<1$ and various values of $\alpha_{30}$, it holds the following.

If condition (14) is satisfied, the system is in free movement state from the initial time unit. And if conditions (15), (19), (20) fulfill, then the system results in free movement state over a finite time.

At fulfilling of conditions (16), (17), (21)

$$
H(0)=3 l-\frac{1}{2}
$$

and over time interval with the duration $\frac{1}{2}+l$ the state vector cyclicly moves to one position to the right, and total cluster delay over this interval equals to $H(0)$.

At fulfilling of conditions (18) clusters form a closed group from time unit $t=1 / 2-2 l$ (at condition $(18) l<1 / 4$ ). Then at time unit $t=1 / 2-2 l$ the delay potential $H(t)$ has value $3 l-\frac{1}{2}$, and after this time the value delay potential of does not change. From time $t=1 / 2-2 l$ the state vector over time interval with duration $\frac{1}{2}+l$ cyclicly moves to one position to the right, and total cluster delay over this interval equals to $H(0)$.

Thus at initial state $\left(l, \frac{1}{2}, \alpha_{30}\right)$, with any $\alpha_{30}$ the state behavior satisfies the condition of Proposition 10.

We have an average cluster velocity

$$
v=1-\frac{H\left(t_{0}\right)}{\frac{1}{2}+l}=1-\frac{3 l-\frac{1}{2}}{3\left(\frac{1}{2}+l\right)}=\frac{4}{3+6 l} .
$$

Let us prove the statement by direct verification of each conditions (13) - (21).

a) Suppose the conditions (13) fulfils. Then in time interval $t \in\left(0, \frac{1}{2}\right)$ all clusters move. At time $t \in\left[0, \frac{1}{2}\right]$

$$
\begin{gathered}
h_{1,2}(t)=h_{2,1}(t)=h_{1,3}(t)=h_{3,1}(t)=h_{3,2}(t)=0, \\
h_{2,3}(t)=l-\alpha_{3,0} .
\end{gathered}
$$

Thus,

$$
H\left(\frac{1}{2}+l-\alpha_{3,0}\right)=0
$$


At moment $t=\frac{1}{2}$ the system results in the state

$$
\alpha_{1}\left(\frac{1}{2}\right)=\frac{1}{2}+l, \alpha_{2}\left(\frac{1}{2}\right)=0, \alpha_{3}\left(\frac{1}{2}\right)=\frac{1}{2}+\alpha_{3,0}
$$

Over time interval $\left(\frac{1}{2}, \frac{1}{2}+l-\alpha_{3,0}\right)$ cluster on contour $C_{2}$ does not move. We have $t \in$ $\left(\frac{1}{2}, \frac{1}{2}+l-\alpha_{3,0}\right)$,

$$
\begin{gathered}
h_{1,2}(t)=h_{2,1}(t)=h_{1,3}(t)=h_{3,1}(t)=h_{3,2}(t)=0, \\
h_{2,3}(t)=l-\alpha_{3,0}-\left(t-\frac{1}{2}\right), \\
h_{1,2}\left(\frac{1}{2}+l-\alpha_{3,0}\right)=h_{2,1}\left(\frac{1}{2}+l-\alpha_{3,0}\right)=h_{1,3}\left(\frac{1}{2}+l-\alpha_{3,0}\right)= \\
=h_{3,1}\left(\frac{1}{2}+l-\alpha_{3,0}\right)=h_{2,3}\left(\frac{1}{2}+l-\alpha_{3,0}\right)= \\
=h_{3,2}\left(\frac{1}{2}+l-\alpha_{3,0}\right)=0, \\
H(t)=\left\{\begin{array}{c}
l-\alpha_{3,0}, 0<t<\frac{1}{2}, \\
l-\alpha_{3,0}-\left(t-\frac{1}{2}\right), \frac{1}{2}<t<\frac{1}{2}+l-\alpha_{3,0}, \\
H\left(\frac{1}{2}+l-\alpha_{3,0}\right)=0 .
\end{array}\right.
\end{gathered}
$$

Therefore, delay potential is constant and equals $l-\alpha_{3,0}$ from initial time unit to time $t=\frac{1}{2}$, then in time interval $t \in\left(\frac{1}{2}, \frac{1}{2}+l-\alpha_{3,0}\right)$ it linear decreases to 0 with velocity 1 . After time unit $\frac{1}{2}+l-\alpha_{3,0}$ the delay potential equals to 0 .

At the time unit $t=\frac{1}{2}+l-\alpha_{3,0}$ the system results in the state

$$
\begin{gathered}
\alpha_{1}\left(\frac{1}{2}+l-\alpha_{3,0}\right)=\frac{1}{2}+2 l-\alpha_{3,0}, \\
\alpha_{2}\left(\frac{1}{2}+l-\alpha_{3,0}\right)=0 \\
\alpha_{3}\left(\frac{1}{2}+l-\alpha_{3,0}\right)=\frac{1}{2}+l
\end{gathered}
$$

that is free movement state.

b) Suppose the conditions (14) fulfils. Then $t \in[0,+\infty)$.

$$
\begin{gathered}
h_{1,2}(t)=h_{2,1}(t)=h_{1,3}(t)=h_{3,1}(t)=h_{2,3}(t)=h_{3,2}(t)=0, \\
H(t) \equiv 0 .
\end{gathered}
$$


Thus, in this case the delay potential equals 0 as at initial time and at any time unit. The system is in free movement state from initial time unit.

c) Suppose that there fulfils either condition (15) or (19). Then in time interval $t \in$ $\left(0,1-\alpha_{3,0}\right)$ all clusters move. At time $t \in\left[0,1-\alpha_{3,0}\right]$

$$
\begin{gathered}
h_{1,2}(t)=h_{2,1}(t)=h_{1,3}(t)=h_{2,3}(t)=h_{3,2}(t)=0, \\
h_{3,1}(t)=\alpha_{3,0}-\frac{1}{2} .
\end{gathered}
$$

At time $t=1-\alpha_{3,0}$ the system results in the state

$$
\alpha_{1}\left(1-\alpha_{3,0}\right)=1+l-\alpha_{3,0}, \alpha_{2}\left(1-\alpha_{3,0}\right)=\frac{3}{2}-\alpha_{3,0}, \alpha_{3}\left(1-\alpha_{3,0}\right)=0 .
$$

In time interval $t \in\left(1-\alpha_{3,0}, \frac{1}{2}\right)$ cluster on contour $C_{3}$ does not move.

We have $t \in\left(1-\alpha_{3,0}, \frac{1}{2}\right)$

$$
\begin{gathered}
h_{1,2}(t)=h_{2,1}(t)=h_{3,1}(t)=h_{2,3}(t)=h_{3,2}(t)=0, \\
h_{1,3}(t)=\alpha_{30}-\frac{1}{2}-\left(t-1+\alpha_{3,0}\right), \\
H(t)=\alpha_{30}-\frac{1}{2}-\left(t-1+\alpha_{3,0}\right) .
\end{gathered}
$$

At time unit $t=\frac{1}{2}$

$$
\begin{gathered}
h_{1,2}\left(\frac{1}{2}\right)=h_{2,1}\left(\frac{1}{2}\right)=h_{1,3}\left(\frac{1}{2}\right)= \\
=h_{3,1}\left(\frac{1}{2}\right)=h_{2,3}\left(\frac{1}{2}\right)=h_{3,2}\left(\frac{1}{2}\right)=0,
\end{gathered}
$$

Thus at initial time unit the delay potential equals to $\alpha_{3,0}-\frac{1}{2}$ and preserves the value until the time unit $1-\alpha_{3,0}$. Then in time interval $t \in\left(1-\alpha_{3,0}, \frac{1}{2}\right)$ delay potential linear decreases with velocity 1 and equals to 0 from time $t=\frac{1}{2}$.

At time $t=\frac{1}{2}$ the system results in the state

$$
\alpha_{1}\left(\frac{1}{2}\right)=\frac{1}{2}+l, \alpha_{2}\left(\frac{1}{2}\right)=0, \alpha_{3}\left(\frac{1}{2}\right)=0,
$$

that is free movement state.

d) Suppose condition (20) fulfils. Then in time interval $t \in\left(0,1-\alpha_{3,0}\right)$ all clusters move. At $t \in\left[0,1-\alpha_{3,0}\right]$

$$
\begin{gathered}
h_{1,2}(t)=h_{2,1}(t)=h_{3,1}(t)=h_{2,3}(t)=0, \\
h_{3,1}(t)=\alpha_{3,0}-\frac{1}{2}
\end{gathered}
$$




$$
\begin{aligned}
& h_{3,2}(t)=\alpha_{3,0}+l-1, \\
& H(t)=2 \alpha_{3,0}+l-\frac{3}{2} .
\end{aligned}
$$

At time unit $t=1-\alpha_{3,0}$ the system results in the state

$$
\alpha_{1}\left(1-\alpha_{3,0}\right)=1+l-\alpha_{3,0}, \alpha_{2}\left(1-\alpha_{3,0}\right)=\frac{3}{2}-\alpha_{3,0}, \alpha_{3}\left(1-\alpha_{3,0}\right)=0 .
$$

In time interval $t \in\left(1-\alpha_{3,0}, \frac{1}{2}\right)$ cluster on contour $C_{3}$ does not move. We have $t \in$ $\left[1-\alpha_{3,0}, \frac{1}{2}\right]$

$$
\begin{gathered}
h_{1,2}(t)=h_{2,1}(t)=h_{3,1}(t)=h_{2,3}(t)=0, \\
h_{3,1}(t)=\alpha_{3,0}-\frac{1}{2}-\left(t-1+\alpha_{3,0}\right) .
\end{gathered}
$$

For potential delay $h_{3,2}(t)$ we have

$$
\begin{gathered}
h_{3,2}(t)=l+\alpha_{3,0}-1-\left(t-1+\alpha_{3,0}\right) \in\left[1-\alpha_{3,0}, l\right], \\
h_{3,2}(t)=0 \in\left(l, \frac{1}{2}\right] \\
H(t)=l+\alpha_{3,0}-1-\left(t-1+\alpha_{3,0}\right) \in\left[1-\alpha_{3,0}, l\right], \\
h_{3,2}(t)=0 \in\left(l, \frac{1}{2}\right] .
\end{gathered}
$$

Therefore

$$
\begin{gathered}
H(t)=2 \alpha_{3,0}+l-\frac{3}{2}-2\left(t-1+\alpha_{3,0}\right), 1-\alpha_{3,0} \leq t \leq l, \\
H(l)=\frac{1}{2}-l \\
H(t)=\frac{1}{2}-l-(t-l), l \leq t \leq \frac{1}{2} .
\end{gathered}
$$

At time $t=\frac{1}{2}$ we have

$$
\begin{gathered}
h_{1,2}\left(\frac{1}{2}\right)=h_{2,1}\left(\frac{1}{2}\right)=h_{1,3}\left(\frac{1}{2}\right)= \\
=h_{3,1}\left(\frac{1}{2}\right)=h_{2,3}\left(\frac{1}{2}\right)=h_{3,2}\left(\frac{1}{2}\right)=0, \\
H\left(\frac{1}{2}\right) \equiv 0 .
\end{gathered}
$$

Thus delay potential equals $2 \alpha_{3,0}+l-\frac{3}{2}$ at initial time and preserves this value until time unit $t=1-\gamma$. After this time the delay potential decreases with velocity 2 to the value 
$\frac{1}{2}-l$ at time unit $t=l$, and in time interval $t \in\left(l, \frac{1}{2}\right)$ it decreases to value 0 , that is preserved at any next time. At time unit $t=\frac{1}{2}$ the system is in the state

$$
\alpha_{1}\left(1-\alpha_{3,0}\right)=\frac{1}{2}+l, \alpha_{2}\left(1-\alpha_{3,0}\right)=0, \alpha_{3}\left(1-\alpha_{3,0}\right)=0
$$

that is free movement state.

e) Suppose there fulfils condition either (16), or (17), or (21). Then in time interval $t \in\left(0, \frac{1}{2}-l\right)$ all clusters move. And at time $t=\frac{1}{2}-l$ the system results in the state

$$
\alpha_{1}\left(\frac{1}{2}-l\right)=\frac{1}{2}, \alpha_{2}\left(\frac{1}{2}-l\right)=1-l, \alpha_{3}\left(\frac{1}{2}-l\right)=\alpha_{3,0}-\frac{1}{2}-l .
$$

At time $t \in\left[0, \frac{1}{2}-l\right]$

$$
\begin{aligned}
h_{1,2}(t)=h_{2,1}(t) & =h_{3,1}(t)=h_{2,3}(t)=0, \\
h_{1,3}(t) & =2 l-\alpha_{3,0}+\frac{1}{2}, \\
h_{3,2}(t) & =\alpha_{3,0}+l-1, \\
H(t) & \equiv 3 l-\frac{1}{2} .
\end{aligned}
$$

In time interval $\left(\frac{1}{2}-l, 1+l-\alpha_{3,0}\right)$ cluster on contour $C_{1}$ does not move. At time $t=$ $1+l-\alpha_{3,0}$ the system results in the state

$$
\alpha_{1}\left(1+l-\alpha_{3,0}\right)=\frac{1}{2}, \alpha_{2}\left(1+l-\alpha_{3,0}\right)=\frac{3}{2}+l-\alpha_{3,0}, \alpha_{3}\left(1+l-\alpha_{3,0}\right)=l .
$$

In time interval $\left(\frac{1}{2}-l, 1+l-\alpha_{3,0}\right)$ cluster on contour $C_{1}$ does not move. In time interval $t \in\left[\frac{1}{2}-l, 1+l-\alpha_{3,0}\right]$

$$
\begin{gathered}
h_{1,2}(t)=h_{3,1}(t)=h_{2,3}(t)=0, \\
h_{1,3}(t)=2 l-\alpha_{30}+\frac{1}{2}-\left(t-\frac{1}{2}+l\right), \\
h_{2,1}(t)=t-\frac{1}{2}+l, \\
h_{3,2}(t)=\alpha_{3,0}+l-1, \\
H(t) \equiv 3 l-\frac{1}{2} .
\end{gathered}
$$

Suppose

$$
\alpha_{3,0}^{\prime}=\frac{3}{2}+l-\alpha_{3,0}
$$

Then

$$
\alpha_{3,0}=\frac{3}{2}+l-\alpha_{3,0}^{\prime} .
$$


We obtain

$$
\frac{1}{2}+l<\alpha_{3,0}^{\prime}<\frac{1}{2}+2 l,
$$

i. e. $\alpha_{30}^{\prime}$ satisfies the condition analogous to the condition for $\alpha_{30}$. We have

$$
\alpha_{3,0}+\alpha_{3,0}^{\prime}=\frac{3}{2}+l
$$

Vector of system state at time $t=1+l-\alpha_{3,0}$ is obtained from vector of initial system state by shifting on one position to the left and substitution of $\alpha_{3,0}$ to $\alpha_{3,0}^{\prime}$ Delay potential equals to

$$
H(t)=3 l-\frac{1}{2}
$$

at initial time unit and reserves constant on any time. At any time unit the value $H(t)$ does not change

$$
H(t) \equiv 3 l-\frac{1}{2}
$$

Thus at time

$$
t=6(1+l)-3\left(\alpha_{3,0}+\alpha_{3,0}^{\prime}\right)=\frac{3}{2}+3 l
$$

the system returns to initial state. Over this time duration each cluster delays twice: first time it delays for $\left(3 l-\alpha_{3,0}\right)$ and second time it delays for $\left(3 l-\alpha_{3,0}^{\prime}\right)$. According to $(22)$, we obtain that total delay of cluster over period equals to $3 l-\frac{1}{2}$ time units.

f) Suppose condition (18) fulfils. In time interval $t \in\left(0, \frac{1}{2}-l\right)$ all clusters move. In time interval $t \in\left(0, \frac{3}{2}-\alpha_{3,0}\right)$ all clusters move. We have

$$
\begin{gathered}
h_{1,2}(t)=h_{2,1}(t)=h_{3,1}(t)=h_{1,3}(t)=h_{2,3}(t)=0, \\
h_{3,2}(t)=\alpha_{3,0}+l-1, \\
H(t) \equiv \alpha_{3,0}+l-1, \\
0 \leq t \leq 1-\alpha_{3,0} .
\end{gathered}
$$

At time $t=\frac{3}{2}-\alpha_{3,0}$ the system results in the state

$$
\alpha_{1}\left(\frac{3}{2}-\alpha_{3,0}\right)=\frac{3}{2}-\alpha_{3,0}+l, \alpha_{2}\left(\frac{3}{2}-\alpha_{3,0}\right)=1-\alpha_{3,0}, \alpha_{3}\left(\frac{3}{2}-\alpha_{3,0}\right)=\frac{1}{2} .
$$

In time interval $t \in\left(\frac{3}{2}-\gamma_{0}, \frac{1}{2}+l\right)$ cluster on contour $C_{3}$ does not move, while other clusters move. The function $h_{3,2}$ decreases in this interval, and beginning from time $t=1-2 l$, function $h_{1,3}$ increases (note that at $\alpha_{3,0}=1+2 l$ ). The equality $\frac{3}{2}-\alpha_{3,0}=$ $1+2 l$ holds, thus, in this case function $h_{1,3}$ increases when time $t=\frac{3}{2}-\alpha_{3,0}$ comes. At $t \in\left[\frac{3}{2}-\alpha_{30}, \frac{1}{2}+l\right]$ we have

$$
h_{1,2}(t)=h_{2,1}(t)=h_{2,3}(t)=h_{3,1}(t)=0,
$$




$$
\begin{gathered}
\left.h_{3,2}(t)=\alpha_{30}+l-1-\left(t-\frac{3}{2}-\alpha_{3,0}\right)\right), \\
h_{1,3}(t)=0, \frac{3}{2}-\alpha_{3,0} \leq t \leq 1-2 l, \\
h_{1,3}(t)=0,1-2 l \leq t \leq \frac{1}{2}+2 l, \\
H(t)=\alpha_{3,0}+l-1-\left(t-\frac{3}{2}-\alpha_{3,0}\right), \\
\frac{3}{2}-\alpha_{3,0} \leq t \leq \frac{1}{2}+l
\end{gathered}
$$

At time $t=\frac{1}{2}+l$ the system results in the state

$$
\alpha_{1}\left(\frac{1}{2}+l\right)=\frac{1}{2}+2 l, \alpha_{2}\left(\frac{1}{2}+l\right)=l, \alpha_{3}\left(\frac{1}{2}+l\right)=\frac{1}{2},
$$

that differs from initial system state by shifting on one position to the right and substitution of $\alpha_{3,0}$ on $1+2 l$, while

$$
\begin{gathered}
h_{1,2}(t)=h_{2,1}(t)=h_{3,1}(t)=h_{1,3}(t)=h_{2,3}(t)=0, \\
h_{3,2}(t)=3 l-\frac{1}{2}, \\
H(t)=3 l-\frac{1}{2} .
\end{gathered}
$$

Thus, in time interval $t \in\left(0, \frac{3}{2}-\alpha_{3,0}\right)$, the function $H(t)$ has value $\alpha_{3,0}+l-1$, then in the time interval $t \in\left(\frac{3}{2}-\alpha_{3,0}, 1-2 l\right)$ function $H(t)$ linear decreases to value $3 l-\frac{1}{2}$, and beginning from time $\frac{1}{2}-2 l$ value of $H(t)$ preserves constant. Next system states return with period

$$
T=\frac{1}{2}+l
$$

Over this time period one of clusters has delay with duration $3 l-\frac{1}{2}$ time units.

All possible cases are considered. Proposition 10 has been proved.

Theorem 2. At value l satisfying the inequality

$$
\frac{1}{6}<l \leq \frac{1}{2}
$$

any admissible initial system state generates a spectral function with one from the two following spectral values 1 and $4 /(3+6 l)$.

Proof. At any value $l<1 / 2$ there exist initial states from which the system results to free movement state after finite time (or the system state is free movement state already). Thus the state is any state of type $\left(\alpha_{1,0}(0), \alpha_{2,0}(0), \alpha_{3,0}(0)\right)$ such that

$$
\alpha_{1,0}(0)=\alpha_{2,0}(0)=\alpha_{3,0}(0) .
$$

Hence and using Proposition 10 the Theorem 2 is proved. 


\section{5. Remarks, generalizations, hypotheses}

\subsection{Behavior of contours trio at $l=\frac{1}{2}$}

In [3] a discrete analog of considered system is studied. There are 2 cells and one particle on each contour of closed chain consisting of $n$ contours. Particle moves at discrete instants of time. If $n=3$ and rule of conflict resolution is conflict left-hand, this system is equivalent to the following case of continuous considered system.

The cluster length on each contour is $\frac{1}{2}$. In the initial state, each cluster is located at a point with coordinate 0 or $\frac{1}{2}$.

Example 1. Suppose that at initial time the system was in the state

$$
\alpha_{1}(0)=l=\frac{1}{2}, \alpha_{2}(0)=l=\frac{1}{2}, \alpha_{3}(0)=\alpha_{3,0} .
$$

If $\alpha_{3,0}=\frac{1}{2}$, then $H(0)=0$, and the system is in free movement state.

If $\alpha_{30}=0$, i. e. initial state is

$$
\alpha_{1}(0)=l=\frac{1}{2}, \alpha_{2}(0)=l=\frac{1}{2}, \alpha_{3}(0)=0,
$$

then

$$
\begin{aligned}
h_{1,2}(0)=h_{2,1}(0)=h_{3,1}(0) & =h_{2,3}(0)=h_{3,2}(0)=0, \\
h_{1,3}(0) & =\frac{1}{2}, \\
H(0) & =\frac{1}{2}
\end{aligned}
$$

and at initial time there is conflict of clusters $C_{1}$ and $C_{3}$. In according of left-priority rule of conflict resolution in time interval $t \in\left(0, \frac{1}{2}\right)$ cluster on contour $C_{1}$ does not move, while clusters on contours $C_{2}$ and $C_{3}$ move. So at time $t=\frac{1}{2}$ the system results in the state

$$
\alpha_{1}\left(\frac{1}{2}\right)=\frac{1}{2}, \alpha_{2}\left(\frac{1}{2}\right)=0, \alpha_{3}\left(\frac{1}{2}\right)=\frac{1}{2},
$$

that is obtained from initial state vector by cyclic shift on one position to the right.

At $t \in\left[0, \frac{1}{2}\right]$ we have

$$
\begin{gathered}
h_{1,2}(t)=h_{2,1}(t)=h_{3,1}(t)=h_{2,3}(t)=0, \\
h_{1,3}(t)=\frac{1}{2}-t, \\
h_{3,2}(t)=t \\
H(t) \equiv \frac{1}{2}
\end{gathered}
$$


and, thus,

$$
\begin{aligned}
h_{1,2}\left(\frac{1}{2}\right)=h_{2,1}\left(\frac{1}{2}\right)=h(1,3)\left(\frac{1}{2}\right) & =h_{3,1}\left(\frac{1}{2}\right)=h_{2,3}\left(\frac{1}{2}\right)=0, \\
h_{3,2}\left(\frac{1}{2}\right) & =\frac{1}{2}, \\
H\left(\frac{1}{2}\right) & =\frac{1}{2},
\end{aligned}
$$

i. e. the potential preserves constant value from initial time to any time unit.

Initial system state repeats over time interval with duration $T=\frac{3}{2}$. Over period the delay of each clusters comes with duration equal to $\frac{1}{2}$.

Average velocity of each clusters equals to

$$
v=\frac{2}{3}
$$

that corresponds to formula

$$
v=\frac{4}{3+6 l}
$$

at $l=\frac{1}{2}$ and results of [1].

\subsection{Behavior of contours trio at $l=\frac{1}{6}$}

In Sect. 2 it was proved that at $l \leq \frac{1}{6}$ the system results to self-organization over finite time .

Example 2. Let cluster length be $l=\frac{1}{6}$ and at time $t=0$ system be in the state

$$
\alpha_{1}(0)=\frac{1}{6}, \alpha_{2}(0)=\frac{1}{2}, \alpha_{3}(0)=\frac{3}{4} .
$$

Then at $t \in\left(0, \frac{1}{3}\right)$

$$
\begin{gathered}
h_{1,2}(t)=h_{2,1}(t)=h_{3,1}(t)=h_{2,3}(t)=h_{3,2}(t)=0, \\
h_{1,3}(t)=\frac{1}{12}-\left(t-\frac{1}{3}\right), \\
H(t)=\frac{1}{12} .
\end{gathered}
$$

In time interval $\left(0, \frac{1}{3}\right)$ all clusters move and in $t=\frac{1}{3}$ the system results in state

$$
\alpha_{1}\left(\frac{1}{3}\right)=\frac{1}{2}, \alpha_{2}\left(\frac{1}{3}\right)=\frac{5}{6}, \alpha_{3}\left(\frac{1}{3}\right)=\frac{1}{12} .
$$


In $\left(\frac{1}{3}, \frac{5}{12}\right)$ cluster on contour $C_{1}$ does not move, and other clusters move. While $t \in\left(\frac{1}{3}, \frac{5}{12}\right)$

$$
\begin{gathered}
h_{1,2}(t)=h_{2,1}(t)=h_{3,1}(t)=h_{2,3}(t)=h_{3,2}(t)=0, \\
h_{1,3}(t)=\frac{1}{12}-\left(t-\frac{1}{3}\right), \\
h_{2,1}(t)=t-\frac{1}{3}, \\
H(t)=\frac{1}{12} .
\end{gathered}
$$

At time $t=\frac{5}{12}$ the system is in state

$$
\begin{gathered}
\alpha_{1}\left(\frac{5}{12}\right)=\frac{1}{2}, \alpha_{2}\left(\frac{5}{12}\right)=\frac{11}{12}, \alpha_{3}\left(\frac{5}{12}\right)=\frac{1}{6}, \\
h_{1,2}\left(\frac{5}{12}\right)=h_{2,1}\left(\frac{5}{12}\right)=h_{1,3}\left(\frac{5}{12}\right)=h_{3,1}\left(\frac{5}{12}\right)=h_{2,3}\left(\frac{5}{12}\right)= \\
=h_{3,2}\left(\frac{5}{12}\right)=0, \\
h_{2,1}\left(\frac{5}{12}\right)=\frac{1}{12}, \\
H(t)=\frac{1}{12} .
\end{gathered}
$$

In time interval $\left(\frac{5}{12}, \frac{1}{2}\right)$ the system is at time $t=\frac{1}{2}$ in state

$$
\alpha_{1}\left(\frac{5}{12}\right)=\frac{7}{12}, \alpha_{2}\left(\frac{5}{12}\right)=0, \alpha_{3}\left(\frac{5}{12}\right)=\frac{1}{4} .
$$

In time interval $\left(\frac{1}{2}, \frac{7}{12}\right)$ cluster on contour $C_{1}$ does not move, while at $t \in\left(\frac{1}{2}, \frac{7}{12}\right)$

$$
\begin{gathered}
h_{1,2}(t)=h_{1,3}(t)=h_{3,1}(t)=h_{2,3}(t)=h_{3,2}(t)=0, \\
h_{2,1}(t)=\frac{1}{12}-\left(t-\frac{1}{2}\right) .
\end{gathered}
$$

We have

$$
\begin{aligned}
& h_{1,2}\left(\frac{7}{12}\right)=h_{2,1}\left(\frac{7}{12}\right)= h_{1,3}\left(\frac{7}{12}\right)=h_{3,1}\left(\frac{7}{12}\right)=h_{2,3}\left(\frac{7}{12}\right)= \\
&= h_{3,2}\left(\frac{7}{12}\right)=0, \\
& H\left(\frac{7}{12}\right)=0 .
\end{aligned}
$$


Thus, delay potential value equals $\frac{1}{12}$ from initial time to time $t=\frac{1}{2}$, and delay potential linear decreases with velocity 1 in time interval $t \in\left(\frac{1}{2}, \frac{7}{12}\right)$ to 0 , and it reserved value 0 in all next time.

At time $t=\frac{7}{12}$ the system is in the state

$$
\alpha_{1}\left(\frac{7}{12}\right)=\frac{7}{12}, \alpha_{2}\left(\frac{7}{12}\right)=0, \alpha_{3}\left(\frac{7}{12}\right)=\frac{1}{4}
$$

that is free movement state.

Thus, according to the above, following is true.

Depending on the value of cluster length $l$ we have

1) If

$$
l \leq \frac{1}{6}
$$

then cluster velocity equals to 1.

2) If

$$
\frac{1}{6}<l<\frac{1}{2}
$$

then, depending on the initial state, the average clusters velocity is equal to 1 or $\frac{4}{3+6 l}$, Fig.3,

3) If

$$
l>\frac{1}{2}
$$

then cluster velocity equals to 0 .

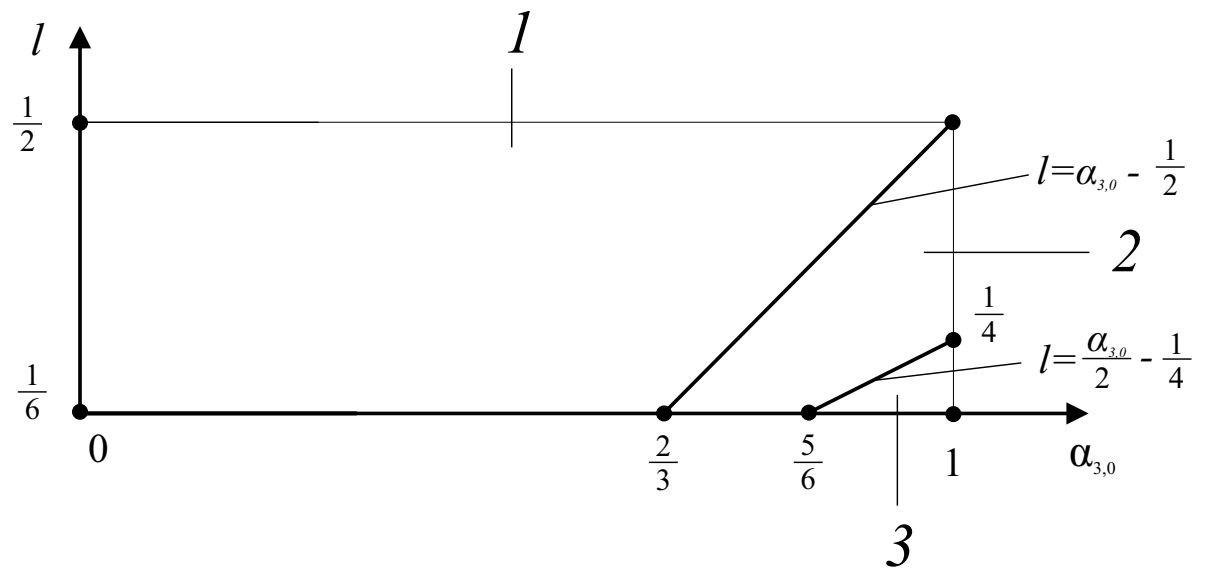

Figure 3: (1) $v=1$, (2) $v=4 /(3+6 l)$, two delays of each cluster per period, (3) $v=4 /(3+6 l)$, one delay 


\subsection{Hypotheses about velocity spectrum for closed chain consisting of arbitrary number of contours}

We formulate hypotheses about the behavior of system that is analogous to considered system, but contour number is arbitrary and equals $n$.

\section{Hypothesis 1.}

1) The system results in free movement state over finite time, if a number $n$ is odd, and the condition

$$
l<\frac{1}{n}
$$

fulfils, or a number $n$ is even and the condition

$$
l<\frac{1}{2 n}
$$

fulfils.

2) For given $n \geq 3$ and $l$ that satisfy the condition

$$
\frac{1}{n}<l \leq \frac{1}{2}
$$

if $n$ is even, or condition

$$
\frac{1}{2 n}<l \leq \frac{1}{2}
$$

if $n$ is odd, in the spectrum of possible values of average velocity for various initial states of the system, except for value 1 , there are no more than $\left[\frac{n}{3}\right]$ values such that less than 1 (square brackets denote the integer part of a number).

These values are calculated as follows.

Let $n$ be even number; $k_{0}$ be maximal natural number $k$ such that

$$
k<n l \text {. }
$$

Then all possible values of average velocity

$$
v=\frac{\frac{n}{2}+k}{\frac{n}{2}+n l},
$$

where $k$ is natural number satisfying the inequalities (23) and

$$
k \geq k_{0}-\left[\frac{n}{3}\right]+1
$$

Let $n$ be odd; $k_{0}$ be maximal natural number $k$ satisfying the following condition

$$
k<n l+\frac{1}{2} .
$$


Then all possible values of average velocity

$$
v=\frac{\frac{n}{2}+k-\frac{1}{2}}{\frac{n}{2}+n l}
$$

where $k$ is natural number satisfying the inequalities (24) and

$$
k \geq k_{0}-\left[\frac{n}{3}\right]+1
$$

\section{The behavior of closed chain with 4 contours}

In the case of closed chain with 4 contours, we have results of computer simulation modeling and formulate the following.

1) If

$$
l \leq \frac{1}{4}
$$

then the system results in a state of self-organization over finite time.

2) If

$$
\frac{1}{4}<l \leq \frac{1}{2}
$$

then, depending on the initial state, the average cluster velocity is equal to 1 or $\frac{3}{2+4 l}$,

3) If

$$
l>\frac{1}{2}
$$

then the system results in a state of collapse.

Thus hypothesis 1 is confirmed.

\section{Conclusion}

A deterministic dynamical system is introduced and considered. It is a closed chain of contours, on which there are movement of clusters with length $l$ in accordance with the specified rules.

It is found that, if $l \leq 1 / 6$, then self-organization takes place. i.e., the system results in free movement state over finite time from any initial state. For $l>1 / 2$, the system results in in collapse state over a finite time. And for any value of $l$, satisfying the condition $1 / 6<l \leq 1 / 2$, the spectrum of possible values of average velocity contains two possible values: 1 and $4 /(3+6 l)$. While what value will average velocity take, depends on the initial system state. We have developed a method to analyze the system behavior. The concept of delay potential is introduced. The properties of delay potential function are studied. In particular, it is proved that the delay potential is a nonincreasing function of time and has a value 0 , when the system results in free movement state. 
Possible generalizations of the results to a closed chain with arbitrary number of contours are considered. Hypothesis about the average clusters velocity and condition of free movement state of the system is discussed.

\section{Acknowledgements}

This work has been supported by the Russian Foundation for Basic Research, Grant No. 17-01-00821-a and No. 17-07-01358-a.

\section{References}

[1] A. P. Buslaev and A. G. Tatashev. Flows on discrete traffic flower. Journal of Mathematics Research, 9(1):98-108, 2017.

[2] A. P. Buslaev, A. G. Tatashev, and M. V. Yashina. Qualitative properties of dynamical system on toroidal chainmail. In T. Simos, G. Psihoyios, and Ch. Tsitouras, editors, 11th International Conference of Numerical Analysis Mathematics.AIP Conference Proceedings 1558., pages 1144-1147, Rhodes, 2013. American Institute of Physics (AIP).

[3] V. V. Kozlov, A. P. Buslaev, and A. G. Tatashev. On synergy of totally connected flows on chainmails. In I.P.Hamilton and J.Vigo-Aquiar, editors, 13th International Conference on Computational and Mathematical Methods in Science and Engineering., pages 861-873, Spain, 2013. University of Almeria.

[4] V. V. Kozlov, A. P. Buslaev, and A. G. Tatashev. Monotonic walks on a necklace and coloured dynamic vector. International Journal of Computer Mathematics, 92(9):19101920, 2014. 